\title{
Chemotherapeutic agent cisplatin suppresses stabilin-1- mediated clearance of EGF by tumor-associated macrophage
}

\author{
Irina Larionova \\ National Research Tomsk State \\ University, \\ Cancer Research Institute, Tomsk \\ National Research Medical Center, \\ Russian Academy of Sciences, \\ Tomsk, Russia \\ larionova0903irina@mail.ru \\ Marina Patysheva \\ National Research Tomsk State \\ University, \\ Cancer Research Institute, Tomsk \\ National Research Medical Center, \\ Russian Academy of Sciences, \\ Tomsk, Russia \\ starin5@ya.ru
}

\author{
Elena Kazakova \\ National Research Tomsk State \\ University \\ Tomsk, Russia \\ kazakova.e.o@mail.ru
}

\author{
Artyem Kiselev \\ Almazov National Medical Research \\ Centre, \\ Saint Petersburg, Russia \\ artem.kiselyov@gmail.com
}

\author{
Julia Kzhyshkowska \\ National Research Tomsk State \\ University, \\ Tomsk, Russia, \\ German Red Cross Blood Service \\ Baden-Württemberg - Hessen, \\ Mannheim, Germany \\ julia.kzhyshkowska@googlemail.com
}

\begin{abstract}
In this study we have demonstrated the suppression of receptor-mediated clearance of tumorsupportive factors EGF by cisplatin in tumor-associated macrophages. These results may be useful for the determining of the mechanisms of the interaction of TAMs and chemotherapy and the mechanism of macrophage-mediated tumor chemoresistance.
\end{abstract}

Keywords - tumor-associated macrophages, cisplatin, EGF, stabilin-1.

\section{Motivation and aim}

Tumor is a complex system of transformed cells interacting with the surrounding heterogeneous cellular and molecular microenvironment. Tumor microenvironment affects key activities of cancer cells and contributes to the tumor response to chemotherapy. Tumor-associated macrophages (TAMs) are a major component of innate immunity supporting primary tumor growth and metastasis [1]. Numerous evidence indicate that TAMs can accumulate in tumors after chemotherapy and contribute to chemoresistance [2]. In this way, it is important to investigate how the functional program of TAMs changes under chemotherapy treatment, and what are the possible mechanisms of the modulating effect of TAMs on the treatment effectiveness. Earlier we have shown that cisplatin may affect the transcriptional program of TAMs, and that in tumors of different localization the effect of the CT agent on the macrophage functions may be different. The aim of this study was to identified the thinner mechanisms of the interaction of TAMs and cisplatin.

\section{Methods}

For the endocytic uptake of EGF we used flow cytometry analysis. Confocal microscopy was used for the analysis of stabilin-1-mediated internalization and endocytic trafficking of EGF in CHO cells and in modeled TAMs differentiated in the presence of conditioned supernatants of breast cancer (MCF-7) and colorectal cancer (Colo206F) cell lines. We performed next-generation sequencing (NGS) of RNA samples obtained from our modeled TAMs. Validation of sequencing data by real-time PCR was performed for selected genes implicated in the endocytic uptake: DNM3, STX8, DENND1A and EHD1.

\section{RESULTS}

For the first time we have demonstrated that stabilin-1 ectopically expressed in $\mathrm{CHO}$ cells mediates endocytic uptake of EGF, key growth factor stimulating progression of breast and colorectal cancer. In the model of primary human TAMs, we have demonstrated that cisplatin decreases stabilin-1mediated internalization and endocytic trafficking of EGF, without significant change in gene expression of scavenger receptor stabilin-1. Molecular mechanisms of cisplatin effects on TAMs were analysed using high throughput RNA sequencing. Gene set enrichment analysis identified that cisplatin contributes to defects in endocyting machinery reducing membrane biogenesis and vesicular transport. Significant suppression of DNM3, STX8, DENND1A and EHD1 genes expression by cisplatin was confirmed by RTPCR. Suppression of receptor-mediated clearance of tumorsupportive factors, such as EGF, by chemotherapeutic drugs may enhance tumor-supporting effect of TAMs creating the microenvironment supporting tumor chemoresistance or relapse after chemotherapy course.

\section{CONCLUSION}

We suggested that suppression of receptor-mediated clearance of tumor-supportive factors by chemotherapeutic drugs may lead to the imbalance in tumor microenvironment and as a result to the chemoresistance.

\section{ACKNOWLEDGMENT}

Supported by the grant RSF No. 19-15-00151.

\section{REFERENCES}

[1] C.E. Lewis, and J.W. Pollard, Cancer Res, vol. 66, pp. 605-612, January 2006.

[2] B.Z. Qian, and J.W. Pollard, Cell, vol. 141, pp. 39-51, April 2010. 